\title{
Gaq gene promoter polymorphisms and rheumatoid arthritis in the Han Chinese population are not associated
}

\author{
Y. Li' , Y. Wang ${ }^{2}$, Y. He ${ }^{1}$, D. Wang ${ }^{1}$, L. Deng ${ }^{1}$, Y. Du ${ }^{3}$ and G. Shi ${ }^{1}$ \\ ${ }^{1}$ State Key Laboratory of Biotherapy, Division of Rheumatology, \\ West China Hospital, Sichuan University, Chengdu, Sichuan, China \\ ${ }^{2}$ Department of Immunology, Chengdu Medical College, Chengdu, \\ Sichuan, China \\ ${ }^{3}$ State Key Laboratory of Biotherapy, GLP Center, \\ West China Hospital, Sichuan University, Chengdu, Sichuan, China \\ Corresponding authors: G. Shi / Y. Du \\ E-mail: shig@scu.edu.cn / duyanchun@yahoo.ca
}

Genet. Mol. Res. 12 (2): 1841-1848 (2013)

Received January 25, 2012

Accepted July 16, 2012

Published January 4, 2013

DOI http://dx.doi.org/10.4238/2013.January.4.6

\begin{abstract}
Mice that lose Gaq from their immune system can spontaneously develop inflammatory arthritis. Gaq expression in the peripheral blood lymphocytes of rheumatoid arthritis (RA) patients is significantly decreased in comparison to that in healthy individuals, and reduced Gaq expression is closely correlated with RA disease activity. These indicate that Gaq plays critical roles in the pathogenesis of RA. To address whether single nucleotide polymorphism in the promoter region of the Gaq gene (GNAQ) influenced Gaq expression in RA patients and was a genetic risk factor for RA, we sequenced the promoter region of GNAQ in a Han Chinese population. A common dinucleotide polymorphism at position -695/-694, an exchange of 2 adjacent nucleotides (GC>TT), was revealed in $118 \mathrm{RA}$ patients and 101 healthy adults. The proportions of genotypes observed for $-695 /-694$ in the RA group were $\mathrm{GC} / \mathrm{GC}$ (65.25\%), GC/TT (33.05\%), and TT/TT $(1.70 \%)$, and those in the control group were GC/GC (62.38\%), GC/TT
\end{abstract}


(33.66\%), and TT/TT (3.96\%). No significant difference in the allele and genotype frequencies between RA patients and healthy controls for dinucleotide polymorphism was found in the Han Chinese population, neither in the whole data set nor in stratified subsets, i.e., rheumatoid factors, anti-cyclic citrullinated peptide antibody, and $\mathrm{G} \alpha \mathrm{q}$ expression status $(\mathrm{P}>0.05)$. We conclude that the $G N A Q$ promoter polymorphism is not a genetic risk factor for RA in the Han Chinese population, and that decreased Gaq expression in peripheral blood lymphocytes of RA might potentially be due to other causes.

Key words: $G N A Q$; Polymorphism; Rheumatoid arthritis; Han Chinese population

\section{INTRODUCTION}

Rheumatoid arthritis (RA), a common autoimmune disease with pathological symptoms of swelling and inflammation, may cause severe damage to diarthrodial joints and even movement disability (Firestein, 2003). This most common inflammatory arthritis affects 0.3 $0.6 \%$ of the population in China (Zheng et al., 2010). Although the exact pathogenesis of the disease is unknown, autoimmunity is critical in the development of RA, and recent progress in defining RA-specific autoantibodies has pointed to the involvement of different immunological pathways in this process, including B, T, and macrophage cell functions (D'Amato et al., 2010).

Heterotrimeric $\mathrm{G}$ proteins play important roles in transmembrane signal transduction, as they transmit signals from the cell surface to the interior of the cell (Wettschureck et al., 2004). Gaq, the $\alpha$ subunit of the Gq protein, belongs to the Gq/11 subfamily of G proteins, and is ubiquitously expressed and involved in various physiological and pathophysiological processes (Wettschureck and Offermanns, 2005; Mizuno and Itoh, 2009). Gaq initially attracted attention in the 1990's for its physiological significance in the cardiovascular system (D'Angelo et al., 1997; Adams et al., 1998). Transgenic mice with cardiac overexpression of Gaq develop cardiac hypertrophy, apoptosis, and heart failure (D'Angelo et al., 1997; Adams et al., 1998; Mende et al., 1998; Sakata et al., 1998). In contrast, ablating the Gaq gene (GNAQ) or dominant $\mathrm{G} \alpha \mathrm{q}$ inhibition prevents reactive hypertrophy and is protective against heart failure (Kina et al., 2000; Wettschureck et al., 2001; Esposito et al., 2002). Similar mechanisms contribute to human left ventricular hypertrophy (LVH). Gaq has also been proven important in metabolic processes. The inhibition of Gq expression by Gq-antisense RNA in the liver and white fat in mice caused obesity and blunted lipolysis, and Gq overexpression increased basal GLUT4 translocation (Galvin-Parton et al., 1997; Imamura et al., 1999; Kanzaki et al., 2000).

Recent studies have demonstrated the function and important roles of $\mathrm{G} \alpha \mathrm{q}$ in immune regulation and autoimmunity. Studies on GNAQ knockout $\left(G n a q^{-1 /}\right)$ mice have indicated that Gnaq $^{-/}$B cells have an intrinsic survival advantage over normal B cells, and the Gnaq $q^{-/}$mice could spontaneously develop autoimmunity against inflammatory arthritis. These data show that the $\mathrm{G} \alpha \mathrm{q}$ protein is critically important for maintaining control of peripheral B cell tolerance induction and repressing autoimmunity (Misra et al., 2010). Our recent data demonstrated that expressions of the Gaq protein and mRNA in peripheral blood lymphocytes (PBLs) of RA patients were significantly decreased compared with those of healthy controls (Wang et al., 
2011). In brief, these results indicated that Gaq expression is involved in the pathogenesis of RA.

Since Gaq expression is implicated in cardiovascular and metabolic disease, the relationships between allelic variations within the $G N A Q$ promoter and these diseases have been studied and certified (Liggett et al., 2007; Frey et al., 2008; Klenke et al., 2010). Allelic variations within the GNAQ promoter that regulate Gaq mRNA expression have not been investigated in RA. Given the importance of Gaq in RA, it is necessary to know the genetic influences of GNAQ promoter polymorphisms in RA. Here, we sequenced $\sim 800 \mathrm{bp}$ of the proximal $5^{\prime}$ region of human $G N A Q$ and analyzed the relationship between polymorphisms in the GNAQ promoter region and RA. In addition, we studied the relationship between different single nucleotide polymorphism (SNP) genotypes/alleles with rheumatoid factor (RF), anti-cyclic citrullinated peptides (anti$\mathrm{CCP}$ ), and the expression levels of Gaq mRNA in the PBLs of RA patients.

\section{MATERIAL AND METHODS}

\section{Subjects}

A total of 118 Han Chinese RA patients who fulfilled the RA classification criteria of the American College of Rheumatology 1987 revised criteria (Arnett et al., 1988) were selected from the outpatient clinic of the Department of Rheumatology, West China Hospital, Sichuan University. The mean age of the population studied was $45.63 \pm 10.38$ years, and the gender ratio was 96 women $(81.36 \%)$ to 22 men (18.64\%). We recruited 101 gender- and age-matched healthy volunteers as controls. The mean age of the control group was $47.09 \pm 9.27$ years, and the gender ratio was 79 women $(78.22 \%)$ to 22 men $(21.78 \%)$. The study was performed in the State Key Laboratory of Biotherapy of Sichuan University after obtaining the approval of the Ethics Committee of West China Hospital and the informed consent of the patients.

\section{DNA extraction}

Blood samples were obtained from the RA patients and healthy controls. Peripheral blood mononuclear cells from heparinized blood samples were isolated by standard density gradient centrifugation over Ficoll-Paque Plus (Axis-Shied PoC AS, Norway). Genomic DNA was extracted using the standard phenol-chloroform method and stored at $-80^{\circ} \mathrm{C}$. The DNA concentration was determined by a NanoDrop ND-1000 spectrophotometer (Thermo Fisher Scientific, USA).

\section{Molecular genetic methods}

Primer pairs derived from a genomic sequence (GenBank accession No. AL160278) were used for amplifying overlapping fragments ( +20 to -514 and -410 to $-798 \mathrm{bp}$ ) of the GNAQ promoter. The following primers were used for amplification and sequencing: GNAQ promoter (-410 to -798 bp) 5'-GGGTCTGGCCCCGACTTCG-3' (sense) and 5'-CCCCCTGCCCCGATT GCCA-3' (anti-sense); (+20 to -514 bp) 5'-CGGATCTGTGCTCCAGTTCAG-3' (sense) and 5'-CGGTGTGAGCGGATAGTCTG-3' (anti-sense). Briefly, GNAQ promoter segments were amplified in a PCR system with a total volume of $25 \mu \mathrm{L}$ PCR mix containing $0.1 \mu$ g genomic 
DNA, $12.5 \mu \mathrm{L} 2 \mathrm{X}$ GC buffer I, $4 \mu \mathrm{L} 2.5 \mathrm{mM}$ of each dNTP mixture, $0.25 \mu \mathrm{L} 5 \mathrm{U} / \mu \mathrm{L}$ Taq polymerase, $0.5 \mu \mathrm{L} 20 \mu \mathrm{mol}$ of each primer, and $\mathrm{ddH}_{2} \mathrm{O}$ sufficient to make the final volume to $25 \mu \mathrm{L}$. The PCR conditions for the upstream fragment consisted of $95^{\circ} \mathrm{C}$ for $5 \mathrm{~min}$, followed by 40 cycles of $95^{\circ} \mathrm{C}$ for $1 \mathrm{~min}, 65^{\circ} \mathrm{C}$ for $30 \mathrm{~s}$, and $72^{\circ} \mathrm{C}$ for $30 \mathrm{~s}$, with a final extension at $72^{\circ} \mathrm{C}$ for $10 \mathrm{~min}$. The PCR conditions for the downstream fragment were as follows: $95^{\circ} \mathrm{C}$ for $5 \mathrm{~min}$, and then 40 cycles were performed at $95^{\circ} \mathrm{C}$ for $30 \mathrm{~s}, 60^{\circ} \mathrm{C}$ for $30 \mathrm{~s}$, and $72^{\circ} \mathrm{C}$ for $30 \mathrm{~s}$, and a final extension thereafter at $72^{\circ} \mathrm{C}$ for $10 \mathrm{~min}$. The products were purified with a PCR purification kit and sequenced using an ABI PRISM 3100 DNA Sequencer (Applied Biosystems, USA).

\section{Reverse-transcription and quantitative PCR}

Total RNA from PBLs was isolated by the TRIzol ${ }^{\mathrm{TM}}$ reagent (Invitrogen, USA) and reverse transcribed according to manufacturer instructions (Takara, Japan). Specific primer sequences (sense/antisense) were as described before (Wang et al., 2011): GAPDH: 5'-GTGAACCATGAGAAGTATGACAAC-3' (sense) and 5'-CATGAGTCCTTCCACGATA CC-3' (antisense), GNAQ: 5'-GTTGATGTGGAGAAGGTGTCTG-3' (sense) and 5'-GTAGGC AGGTAGGCAGGGT-3' (antisense). A 25- $\mu$ L SYBR Green II PCR mixture was used, containing $2 \mu \mathrm{L}$ cDNA, $1 \mu \mathrm{L}$ forward primer, $1 \mu \mathrm{L}$ reverse primer, and $12.5 \mu \mathrm{L}$ SYBR master mix (Takara). Quantitative PCR was performed with the $\mathrm{iQ}^{\mathrm{TM}} 5$ and $\mathrm{MyiQ}^{\mathrm{TM}}$ RealTime PCR Detection Systems (Bio-Rad, USA). mRNA expression was normalized to $G A P D H$ expression levels and relative expression was calculated with the $2^{-\Delta \Delta \mathrm{Ct}}$ method (Schmittgen and Livak, 2008).

\section{Statistical analysis}

Statistical analyses were performed with the SPSS software (version 19.0, SPSS Inc., USA). The allele and genotype frequencies were calculated by direct count. The chisquare $\left(\chi^{2}\right)$ test was used to compare allele/genotype frequencies and clinical characteristics between groups, and the Hardy-Weinberg equilibrium (HWE) of genotype distribution. Odds ratio (OR) and $95 \%$ confidence intervals $(95 \% \mathrm{CI})$ were calculated using logistic regression. Data of Gaq expression are reported as means $\pm \mathrm{SD}$. P values $<0.05$ were considered significant.

\section{RESULTS}

To identify naturally occurring genetic variations of the GNAQ promoter, we amplified and sequenced the flanking region of the GNAQ gene extending to approximately $\sim 800 \mathrm{bp}$ in the $5^{\prime}$ direction of the transcription initiation site. A dinucleotide variation was identified at -695/-694 (GC/TT). The genotype distribution of the polymorphisms GC(-695/-694)TT was in HWE for patients $(\mathrm{P}=0.24)$ and deviated from HWE for controls $(\mathrm{P}=0.82)$. Table 1 lists the genotype and allele distribution of the GC(-695/-694)TT polymorphisms in patients and controls. There was no significant difference between the 2 groups regarding allele distribution $\left[\chi^{2}=0.46 ; \mathrm{P}=0.50 ; \mathrm{OR}=\right.$ 1.18: $(95 \% \mathrm{CI}=0.71-1.95)]$. No differences in genotype distribution for the dinucleotide variation between patients and controls were detected $\left(\chi^{2}=1.10, \mathrm{P}=0.58\right)$. 


\begin{tabular}{|c|c|c|c|c|c|c|}
\hline Polymorphism & Patients [N (\%)] & Controls [N (\%)] & $\chi^{2}$ & $P$ & OR & $95 \% \mathrm{CI}$ \\
\hline \multicolumn{7}{|l|}{ Genotype } \\
\hline GC/GC & $77(65.25 \%)$ & $63(62.38 \%)$ & 1.10 & 0.58 & & \\
\hline GC/TT & $39(33.05 \%)$ & $34(33.66 \%)$ & & & & \\
\hline TT/TT & $2(1.70 \%)$ & $4(3.96 \%)$ & & & & \\
\hline \multicolumn{7}{|l|}{ Allele } \\
\hline $\mathrm{GC}$ & $193(81.78 \%)$ & $160(79.21 \%)$ & 0.46 & 0.50 & 1.18 & $0.71-1.95$ \\
\hline TT & $43(18.22 \%)$ & $42(20.79 \%)$ & & & & \\
\hline
\end{tabular}

$\mathrm{OR}=$ odds ratio $; 95 \% \mathrm{CI}=95 \%$ confidence interval.

To determine whether the GC(-695/-694)TT polymorphism at the GNAQ promoter region might influence the clinical spectrum of RA, we stratified the RA patients according to positive or negative findings for RF and anti-CCP. Of the RA patients, 94.07\% (111/118) were RF-positive and $98.31 \%$ (116/118) were anti-CCP antibody-positive. According to the anti-CCP titer, the anti-CCP group was further divided into another 2 subgroups: $5<$ anti-CCP $<100$ and anti-CCP $>100$.

As shown in Table 2, the GC haplotype was present in the RF-negative subgroup at higher frequencies than that in the RF-positive subgroup ( 85.71 and $81.53 \%$, respectively), but we did not find a significant difference between the groups $(\mathrm{P}=0.69)$. Regarding stratification for anti-CCP status, no difference in allele or genotype frequencies between the $5<$ anti-CCP $<100$ and anti-CCP $>100$ groups was found (Table 3).

Table 2. Comparison of GNAQ gene polymorphisms between rheumatoid factor-positive ( $\mathrm{RF}+$ ) and -negative (RF-) rheumatoid arthritis patients.

\begin{tabular}{|c|c|c|c|c|c|c|}
\hline Polymorphism & $\mathrm{RF}+[\mathrm{N}=111(\%)]$ & RF- $[\mathrm{N}=7(\%)]$ & $\chi^{2}$ & $\mathrm{P}$ & OR & $95 \% \mathrm{CI}$ \\
\hline \multicolumn{7}{|l|}{ Genotype } \\
\hline $\mathrm{GC} / \mathrm{GC}$ & $72(64.86 \%)$ & $5(71.43 \%)$ & 0.21 & 0.90 & & \\
\hline $\mathrm{GC} / \mathrm{TT}$ & $37(33.33 \%)$ & $2(28.57 \%)$ & & & & \\
\hline $\mathrm{TT} / \mathrm{TT}$ & $2(1.81 \%)$ & $0(0 \%)$ & & & & \\
\hline \multicolumn{7}{|l|}{ Allele } \\
\hline $\mathrm{GC}$ & $181(81.53 \%)$ & $12(85.71 \%)$ & 0.15 & 0.69 & 0.74 & $0.08-3.51$ \\
\hline TT & $41(18.47 \%)$ & $2(14.29 \%)$ & & & & \\
\hline
\end{tabular}

For abbreviations, see legends to Table 1.

Table 3. Comparison of GNAQ gene polymorphisms between rheumatoid arthritis patients with different titers of anti-cyclic citrullinated peptides (anti-CCP).

\begin{tabular}{|c|c|c|c|c|c|c|}
\hline Polymorphism & $5<$ anti-CCP $<100[\mathrm{~N}=31(\%)]$ & Anti-CCP $>100[\mathrm{~N}=85(\%)]$ & $\chi^{2}$ & $\mathrm{P}$ & OR & $95 \% \mathrm{CI}$ \\
\hline \multicolumn{7}{|l|}{ Genotype } \\
\hline $\mathrm{GC} / \mathrm{GC}$ & $21(67.74 \%)$ & $56(66.18 \%)$ & 0.67 & 0.71 & & \\
\hline GC/TT & $9(29.03 \%)$ & $28(32.35 \%)$ & & & & \\
\hline TT/TT & $1(3.23 \%)$ & $1(1.47 \%)$ & & & & \\
\hline \multicolumn{7}{|l|}{ Allele } \\
\hline GC & $51(82.26 \%)$ & $140(82.35 \%)$ & 0 & 0.99 & 0.99 & $0.44-2.37$ \\
\hline TT & $11(17.74 \%)$ & $30(17.65 \%)$ & & & & \\
\hline
\end{tabular}

For abbreviations, see legends to Table 1. 
We measured Gaq mRNA expression by quantitative real-time PCR. Consistent with our published data (Wang et al., 2011), Gaq expression at mRNA level in the PBLs from RA patients was significantly decreased in comparison to that in healthy individuals $(\mathrm{P}<0.05)$. However, Gaq mRNA expression in RA between different SNP genotypes/alleles was not significantly different $(\mathrm{P}=0.47)$ (Table 4$)$.

Table 4. Expression of Gaq in mRNA level in individuals with different genotypes.
\begin{tabular}{lcccc}
\hline Genotype & Patients No. & mRNA expression & F & P \\
\hline GC/GC & 77 & $0.79 \pm 0.18$ & 0.76 & 0.47 \\
GC/TT & 39 & $0.80 \pm 0.21$ & & \\
TT/TT & 2 & $0.75 \pm 0.15$ & & \\
\hline
\end{tabular}

\section{DISCUSSION}

The precise etiology of RA remains poorly understood; however, evidence suggests that genetics contributes to susceptibility to RA (Orozco et al., 2006). An association between RA risk and the major genetic susceptibility locus, human leukocyte antigen (HLA), has been established, but HLA is thought to contribute less than one-third of the total genetic susceptibility component (Deighton et al., 1989; Wordsworth et al., 1989), and several other non-HLA genes (such as TLR-4, PTPN22) (Kilding et al., 2003; Begovich et al., 2004; Radstake et al., 2004; Lee et al., 2005; Orozco et al., 2005) may be involved in the susceptibility to RA. When we considered the important roles of Gaq in autoimmunity and decreased expression of Gaq in the PBLs from RA patients (Misra et al., 2010; Wang et al., 2011), we considered the $G N A Q$ promoter region a convincing candidate gene for genetic association studies investigating susceptibility for RA. In this study, we sequenced the GNAQ promoter using a Chinese cohort of Asian ethnicity and analyzed the association between RA and $G N A Q$ promoter polymorphism. We detected GC(-695/-694)TT within the promoter region in the samples. The dinucleotide polymorphism was described as having -695/-694 TT allele frequency of 0.467 or 0.48 in Caucasians and 0.329 in African Americans in previous studies (Liggett et al., 2007; Frey et al., 2008; Klenke et al., 2010). Comprising 118 RA and 101 healthy controls from the Han Chinese population, the allele frequency of the -695/-694 TT polymorphism was 0.18 in RA patients and 0.21 in controls. The G(-168)A and G(-173)A variants have been identified in other reports, but the allele frequencies were different (Liggett et al., 2007; Frey et al., 2008; Klenke et al., 2010). Frey et al. (2008) reported that allele frequencies of the G(-173)A and G(-168)A polymorphisms were rare $(<0.05)$, and Liggett et al. (2007) reported allele frequencies of $>0.05$. In another report, the minor allele frequencies for the $\mathrm{A}(-173)$ and $\mathrm{A}(-168)$ alleles were 0.045 and 0.079 , respectively. It is noteworthy that G(-168)A was relevant and was proven to be in linkage with GC(-695/-694)TT in the study (Klenke et al., 2010). We did not identify the polymorphisms at the -173 and -168 sites. No other polymorphism was detected in all RA cases and controls. The distribution of these SNPs in $G N A Q$ in this Chinese population was quite different from that in the West. To a certain extent, the genetic background of the studied populations could explain the lack of agreement between published GNAQ polymorphism genetic association studies.

The role of the GNAQ promoter polymorphism in disease initially attracted attention for its association with accelerated mortality in African-American heart failure, which 
was reported in an American study (Liggett et al., 2007). They demonstrated that GC/ TT substitution eliminated Sp-1 binding to this promoter fragment. Thus, the GNAQ -695/694 promoter polymorphism alters transcription factor binding, increases promoter activity, and reduces survival time in African-American heart failure, but not in Caucasians (Liggett et al., 2007). Another study on cardiac hypertrophy also referred to the association between the GNAQ promoter region polymorphism and enhanced promoter activity, Gq expression, intracellular signal transduction, and increased prevalence of LVH, particularly in women (Frey et al., 2008). Klenke et al. (2010) hypothesized and characterized the association of GNAQ promoter diplotypes with insulin resistance and obesity in polycystic ovary syndrome. They found that the A(-168) allele is functionally relevant and in linkage with GC(-695/-694)TT, and associated with decreased transcriptional activity and altered transcription factor binding. Given the importance of Gaq in autoimmunity and its decreased expression in the PBLs of RA (Misra et al., 2010; Wang et al., 2011), polymorphisms within the GNAQ promoter might be associated with RA and might be a cause of decreased Gaq expression in the PBLs of RA patients. We sequenced the GNAQ promoter and identified a dinucleotide variation at -695/694(GC/TT), and studied the association between this and RA in a Han Chinese population.

Unfortunately, we found no significant difference between patients and controls by allele or genotype status. The results suggest that the dinucleotide polymorphism GC(-695/-694)TT of GNAQ is not a significant risk factor for the development of RA in the Han Chinese population. This may be due to ethnic variation or the small sample size, resulting in low statistical power in this study that rendered it unable to identify the effect of this SNP on RA. This issue and the question of whether GNAQ promoter polymorphisms can impact RA will require further study in other genetic cohorts. Our results also indicate that GNAQ promoter polymorphisms may not be a cause of the decreased Gaq expression in the PBLs of RA. We speculate that the altered expression of Gaq PBLs of RA may be caused by epigenetic regulation (such as DNA methyltransferase and histone deacetylases). The epigenetic regulation of Gaq in the PBLs of RA is under investigation.

\section{ACKNOWLEDGMENTS}

Research supported by the National Natural Science Foundation of China (Grants \#30830094, \#30972678) to Dr. Guixiu Shi.

\section{REFERENCES}

Adams JW, Sakata Y, Davis MG, Sah VP, et al. (1998). Enhanced Galphaq signaling: a common pathway mediates cardiac hypertrophy and apoptotic heart failure. Proc. Natl. Acad. Sci. U. S. A. 95: 10140-10145.

Arnett FC, Edworthy SM, Bloch DA, McShane DJ, et al. (1988). The American Rheumatism Association 1987 revised criteria for the classification of rheumatoid arthritis. Arthritis Rheum. 31: 315-324.

Begovich AB, Carlton VE, Honigberg LA, Schrodi SJ, et al. (2004). A missense single-nucleotide polymorphism in a gene encoding a protein tyrosine phosphatase (PTPN22) is associated with rheumatoid arthritis. Am. J. Hum. Genet. 75: 330-337.

D’Amato M, Zucchelli M, Seddighzadeh M, Anedda F, et al. (2010). Analysis of neuropeptide S receptor gene (NPSR1) polymorphism in rheumatoid arthritis. PLoS One 5: e9315.

D’Angelo DD, Sakata Y, Lorenz JN, Boivin GP, et al. (1997). Transgenic Galphaq overexpression induces cardiac contractile failure in mice. Proc. Natl. Acad. Sci. U. S. A. 94: 8121-8126.

Deighton CM, Walker DJ, Griffiths ID and Roberts DF (1989). The contribution of HLA to rheumatoid arthritis. Clin. Genet. 36: 178-182. 
Esposito G, Rapacciuolo A, Naga Prasad SV, Takaoka H, et al. (2002). Genetic alterations that inhibit in vivo pressureoverload hypertrophy prevent cardiac dysfunction despite increased wall stress. Circulation 105: 85-92.

Firestein GS (2003). Evolving concepts of rheumatoid arthritis. Nature 423: 356-361.

Frey UH, Lieb W, Erdmann J, Savidou D, et al. (2008). Characterization of the GNAQ promoter and association of increased Gq expression with cardiac hypertrophy in humans. Eur. Heart J. 29: 888-897.

Galvin-Parton PA, Chen X, Moxham CM and Malbon CC (1997). Induction of Galphaq-specific antisense RNA in vivo causes increased body mass and hyperadiposity. J. Biol. Chem. 272: 4335-4341.

Imamura T, Vollenweider P, Egawa K, Clodi M, et al. (1999). G alpha-q/11 protein plays a key role in insulin-induced glucose transport in 3T3-L1 adipocytes. Mol. Cell Biol. 19: 6765-6774.

Kanzaki M, Watson RT, Artemyev NO and Pessin JE (2000). The trimeric GTP-binding protein (G(q)/G(11)) alpha subunit is required for insulin-stimulated GLUT4 translocation in 3T3L1 adipocytes. J. Biol. Chem. 275: 7167-7175.

Kilding R, Akil M, Till S, Amos R, et al. (2003). A biologically important single nucleotide polymorphism within the tolllike receptor-4 gene is not associated with rheumatoid arthritis. Clin. Exp. Rheumatol. 21: 340-342.

Kina T, Ikuta K, Takayama E, Wada K, et al. (2000). The monoclonal antibody TER-119 recognizes a molecule associated with glycophorin A and specifically marks the late stages of murine erythroid lineage. Br. J. Haematol. 109: 280-287.

Klenke S, Tan S, Hahn S, Mann K, et al. (2010). A functional GNAQ promoter haplotype is associated with altered $\mathrm{Gq}$ expression and with insulin resistance and obesity in women with polycystic ovary syndrome. Pharmacogenet. Genomics 20: 476-484.

Lee AT, Li W, Liew A, Bombardier C, et al. (2005). The PTPN22 R620W polymorphism associates with RF positive rheumatoid arthritis in a dose-dependent manner but not with HLA-SE status. Genes Immun. 6: 129-133.

Liggett SB, Kelly RJ, Parekh RR, Matkovich SJ, et al. (2007). A functional polymorphism of the Galphaq (GNAQ) gene is associated with accelerated mortality in African-American heart failure. Hum. Mol. Genet. 16: 2740-2750.

Mende U, Kagen A, Cohen A, Aramburu J, et al. (1998). Transient cardiac expression of constitutively active Galphaq leads to hypertrophy and dilated cardiomyopathy by calcineurin-dependent and independent pathways. Proc. Natl. Acad. Sci. U. S. A. 95: 13893-13898.

Misra RS, Shi G, Moreno-Garcia ME, Thankappan A, et al. (2010). G alpha q-containing G proteins regulate B cell selection and survival and are required to prevent B cell-dependent autoimmunity. J. Exp. Med. 207: 1775-1789.

Mizuno N and Itoh H (2009). Functions and regulatory mechanisms of Gq-signaling pathways. Neurosignals 17: 42-54.

Orozco G, Sanchez E, Gonzalez-Gay MA, Lopez-Nevot MA, et al. (2005). Association of a functional single-nucleotide polymorphism of PTPN22, encoding lymphoid protein phosphatase, with rheumatoid arthritis and systemic lupus erythematosus. Arthritis Rheum. 52: 219-224.

Orozco G, Rueda B and Martin J (2006). Genetic basis of rheumatoid arthritis. Biomed. Pharmacother. 60: 656-662.

Radstake TR, Franke B, Hanssen S, Netea MG, et al. (2004). The Toll-like receptor 4 Asp299Gly functional variant is associated with decreased rheumatoid arthritis disease susceptibility but does not influence disease severity and/or outcome. Arthritis Rheum. 50: 999-1001.

Sakata Y, Hoit BD, Liggett SB, Walsh RA, et al. (1998). Decompensation of pressure-overload hypertrophy in G alpha q-overexpressing mice. Circulation 97: 1488-1495.

Schmittgen TD and Livak KJ (2008). Analyzing real-time PCR data by the comparative C(T) method. Nat. Protoc. 3: 1101-1108.

Wang Y, Li Y, He Y, Sun Y, et al. (2011). Expression of G protein $\alpha$ q subunit is decreased in lymphocytes from rheumatoid arthritis patients and is correlated with disease activity. Scand. J. Immunol. (Epub ahead of print).

Wettschureck N and Offermanns S (2005). Mammalian G proteins and their cell type specific functions. Physiol. Rev. 85: 1159-1204.

Wettschureck N, Rutten H, Zywietz A, Gehring D, et al. (2001). Absence of pressure overload induced myocardial hypertrophy after conditional inactivation of Galphaq/Galpha11 in cardiomyocytes. Nat. Med. 7: 1236-1240.

Wettschureck N, Moers A and Offermanns S (2004). Mouse models to study G-protein-mediated signaling. Pharmacol. Ther. 101: 75-89.

Wordsworth BP, Lanchbury JS, Sakkas LI, Welsh KI, et al. (1989). HLA-DR4 subtype frequencies in rheumatoid arthritis indicate that DRB1 is the major susceptibility locus within the HLA class II region. Proc. Natl. Acad. Sci. U. S. A. 86: 10049-10053.

Zheng B, Li Q, Wei C, Qin J, et al. (2010). Lack of association of TLR4 gene Asp299Gly and Thr399Ile polymorphisms with rheumatoid arthritis in Chinese Han population of Yunnan Province. Rheumatol. Int. 30: 1249-1252. 